\title{
Moment-to-moment interaction between affectivity and coping behaviours in bipolar disorder and the role of cognitive appraisals
}

Michelle Hoi-ting Leung, Suzanne Ho-wai So, Nate Tsz-Kit Kwok, Iris Hoi-ching Ng, Pui-shuen Chan, Chloe Chor-wing Lo, Shirley Na, Arthur Dun-ping Mak and Sing Lee

\section{Background}

Individuals with bipolar disorder respond to affective symptoms with a range of coping behaviours, which may further maintain the symptoms

\section{Aims}

To examine moment-to-moment dynamics between affective states and coping behaviours, and to evaluate the role of cognitive appraisals of internal states as moderators.

\section{Method}

Forty-six individuals with bipolar disorder completed a clinical interview and an experience sampling assessment over 6 days Time-lagged analyses were conducted by multilevel regression modelling.

\section{Results}

A total of 1807 momentary entries were analysed. Negative affect predicted an increase in rumination at the subsequent time point ( $\beta=0.21$, s.e. $=0.08, P=0.009,95 \% \mathrm{Cl} 0.05-0.36$ ), and vice versa $(\beta=0.03$, s.e. $=0.01, P=0.009,95 \% \mathrm{Cl} 0.01-0.05)$. Positive affect predicted an increase in adaptive coping ( $\beta=0.26$ s.e. $=0.11, P=0.018,95 \% \mathrm{Cl} 0.04-0.47)$, and vice versa $(\beta=0.02$, s.e. $=0.01, P=0.019,95 \% \mathrm{Cl} 0.00-0.03)$. Positive affect also predicted a decrease in rumination $(\beta=-0.15$, s.e. $=0.06$,
$P=0.014,95 \% \mathrm{Cl}-0.26$ to -0.03$)$, and vice versa $(\beta=-0.03$, s.e. $=0.01, P=0.016,95 \% \mathrm{Cl}-0.06$ to -0.01$)$. Extreme cognitive appraisals predicted stronger associations between affective states and coping behaviours.

\section{Conclusions}

Feedback loops between affective states and coping behaviours were revealed in the daily life of individuals with bipolar disorder which were moderated by extreme cognitive appraisals.

\section{Declaration of interest}

None.

\section{Keywords}

Bipolar disorder; affect; coping; experience sampling; cognitive appraisals.

\section{Copyright and usage}

(C) The Royal College of Psychiatrists 2019. This is an Open Access article, distributed under the terms of the Creative Commons Attribution licence (http://creativecommons.org/licenses/by/ 4.0/), which permits unrestricted re-use, distribution, and reproduction in any medium, provided the original work is properly cited.
Bipolar disorder is characterised by instability of affect within and between episodes. There has been an increase in interest in understanding how affective symptoms are maintained by an individual's behaviours. Researchers have suggested that individuals with bipolar disorder engage in coping behaviours in an attempt to control or regulate their symptoms, with maladaptive coping behaviours in turn exacerbating the affective symptoms. ${ }^{1}$

\section{Relationship between affective states and coping behaviours}

The association between affective states and coping behaviour has recently been tested using experience sampling methodology (ESM). In Pavlickova et $a l^{2}$ level of negative affect was found to predict an increase in rumination at the next assessment time point, whereas engagement in rumination predicted a subsequent increase in negative affect. On the contrary, positive affect was found to predict an increase in risk-taking behaviours, and risktaking behaviours in turn increased positive affect. In two other experience sampling studies involving non-patient samples, Brans et $a l^{3}$ also reported that rumination was associated with increases in negative affect and decreases in positive affect. However, Pavlickova $e t ~ a l^{2}$ s finding of the association between positive affect and risk-taking behaviours has not been replicated. It is of note that in Pavlickova et $a l,{ }^{2}$ the positive affect factor consisted of the following items: cheerful, excited, relaxed and satisfied. It is not clear whether these items represented elevated affect analogous to mania/hypomania (such as excessive excitement and arousal) or a pleasant and calm emotional state. Neurophysiological studies ${ }^{4,5}$ have suggested that elevated affect and positive affect are two distinct dimensions, with the former relating to drive and arousal that underpins activated and energised behaviours, and the latter relating to a sense of contentment, well-being and peacefulness when one is not actively seeking out resources. When positive affect consisted of being happy and relaxed (without the excitement and arousal component), Brans et $a l^{3}$ reported that adaptive coping strategies (such as reflection, distraction and social sharing) were associated with increases in positive affect. Therefore, it remains unclear whether positive affect (or its specific components) is more closely associated with adaptive coping or risk-taking. As elevated affect and positive affect are not positively correlated, but may be experienced simultaneously by individuals with bipolar disorder, ${ }^{6}$ understanding the relationship between affect and coping behaviour would be advanced if we assess positive affect and elevated affect separately as patients with bipolar disorder experience their daily lives. Therefore, the current study will test the moment-to-moment associations between a range of affective states (negative affect, positive affect and elevated affect) and coping behaviours (rumination, adaptive coping and risk-taking).

\section{Role of cognitive appraisals as moderator}

Individuals with bipolar disorder have a tendency to appraise activated internal states as extremely positive or negative. ${ }^{1}$ It was 
suggested that positive appraisals of internal states predicted activation via engagement in ascent behaviour (such as risk-taking), and that negative appraisals of internal states predicted engagement in descent behaviour (such as rumination). ${ }^{7}$ However, the role of cognitive appraisals on moment-to-moment interactions between affective states and coping behaviour has not been directly tested.

\section{Aim of study}

The present study aimed to examine the moment-to-moment associations between affective states and coping behaviour, and the effect of baseline level of extreme cognitive appraisals on these moment-to-moment relationships. Key hypotheses were as follows:

(a) Momentary level of affective states will drive changes in coping behaviour at the next assessment time point:

(i) Level of negative affect at time $t$ will predict an increase in rumination at time $t+1$,

(ii) Level of positive affect at time $t$ will predict an increase in adaptive coping at time $t+1$,

(iii) Level of elevated affect at time $t$ will predict an increase in risk taking at time $t+1$;

(b) Momentary engagement in coping behaviour will drive changes in affective states at the next assessment time point:

(i) Level of rumination at time $t$ will predict an increase in negative affect at time $t+1$,

(ii) Level of adaptive coping at time $t$ will predict an increase in positive affect at time $t+1$,

(iii) Level of risk taking at time $t$ will predict an increase in elevated affect at time $t+1$;

(c) Baseline level of extreme cognitive appraisals will moderate the moment-to-moment relationship between affective states and coping behaviours. Specifically, extreme cognition will predict stronger associations between negative affect and rumination, and between elevated affect and risk-taking.

\section{Method}

\section{Sample}

Ethical approval for the study was granted by the Joint Chinese University of Hong Kong and New Territories East Cluster Clinical Research Ethics Committee (CRE-2013-652-T). Inclusion criteria were: outpatients with a diagnosis of bipolar spectrum disorder, age 18 years or above and an ability to read Chinese and to complete self-report measures independently. Exclusion criteria were intellectual disability, brain injury and a primary diagnosis of substance-related disorder or organic bipolar disorder.

\section{Measures}

ESM

ESM is a diary method that collects participants' self-reports at various moments throughout the day and has been applied in examining psychopathology among individuals with bipolar disorder. ${ }^{2,8}$ In our current study, moment-to-moment levels of affective symptoms and coping behaviours were assessed by an assessment app on an electronic device. The app (named 'Questionnaire') was designed and developed by the Clinical Psychology Laboratory of the Chinese University of Hong Kong, and has been used in other studies. ${ }^{9}$ The Questionnaire app can be downloaded through the iOS and Android platforms. Consistent with other ESM studies, ${ }^{2,10,11}$ our app generated ten moments each day, over 6 consecutive days. The ESM signal was emitted at quasi-random intervals during the day $(10.00-22.00 \mathrm{~h})$. Care was taken so that the ESM assessment captured participants' flow of daily life without disrupting their routine. Upon communication with individual patients, we made adjustment to the signalling schedule according to their daily routine and sleep hours, as appropriate.

With reference to previous ESM studies, ${ }^{12,13}$ three items were included for negative affect (irritated, low and tense) and positive affect (cheerful, relaxed and content), respectively. In addition, we included three new items to measure elevated affect (excited, elevated and energetic). These items were assessed on a seven-point Likert scale (from 1, 'not at all', to 7, 'very much'). We reported acceptable to excellent internal consistency for each set of affective items (Cronbach's $\alpha=0.82$ for negative affect, 0.92 for positive affect, and 0.72 for elevated affect).

Based on a revised version of the Response Style Questionnaire, ${ }^{14}$ a total of ten ESM items (see Appendix) were included to assess participants' use of a range of coping strategies, including rumination, distraction, problem-solving and risktaking. Following a previous study, 'distraction' and 'problemsolving' were grouped under 'adaptive coping'. These three coping components (rumination, risk-taking and adaptive coping) yielded satisfactory levels of internal consistency in our study (Cronbach's $\alpha=0.63-0.92$ ).

\section{Clinical interview}

All participants completed a clinical assessment interview at baseline. Psychiatric diagnosis was determined using the Chinese-bilingual Structured Clinical Interview for DSM-IV (Axis I, Patient version). ${ }^{15}$ Depressive symptoms, manic symptoms and anxiety symptoms were assessed by the ten-item Montgomery-Asberg depression rating scale (MADRS), ${ }^{16}$ the 11-item Young Mania Rating Scale (YMRS) ${ }^{17}$ and the 14-item Hamilton Rating Scale for Anxiety (HRSA), ${ }^{18}$ respectively. The score range was $0-60$ for the MADRS and YMRS, and 0-56 for the HRSA.

\section{Cognitive appraisals of changes in internal states}

The Hypomanic Attitudes and Positive Predictions Inventory (HAPPI) is a 26-item self-report inventory that was designed to measure extreme and personalised positive and negative appraisal style of internal states for individuals with bipolar disorder. ${ }^{19}$ It was validated in patients with bipolar disorder and nonclinical control samples. ${ }^{1}$ The HAPPI yielded five factor scores: catastrophic beliefs about internal states, reduced social regulation, an activating response style, success activation and triumph over fear, and loss of control when activated. HAPPI has been translated into Chinese for this study. Graduate-level students conducted the translation and back-translation procedures independently. Any discrepancy was resolved by discussion. The translated version of the HAPPI showed excellent internal consistencies in the current sample (Cronbach's $\alpha$ for the factor scores ranging from 0.91 to $0.97)$.

\section{Procedure}

Participants were either referred by clinicians or volunteered to take part in this study. Their suitability for study participation was confirmed by their psychiatrists. All participants provided written consent before the start of the study. At the end of the baseline interview, participants completed a set of questionnaires and were guided through the use of the ESM app individually. Over the following 6 days, participants completed the ESM on their own mobile phone or on an iPod Touch borrowed from our laboratory. To increase compliance to the assessment, participants were contacted at least twice during the 6-day period; those 
who had difficulty in using the app received further support and training.

\section{Statistical analysis}

Statistical analysis was carried out using hierarchical linear modelling, a statistical approach that accounts for within person (level 1) and between-person (level 2) variance. Multilevel regression modelling with maximum likelihood estimation was used to model the relationships between momentary variables, with the assumption that data are missing at random. ${ }^{20}$ To model between-moment associations within the same day, new variables for each lagged level-1 variable at its subsequent moment was named $t+1$. We modelled the effect of the level-1 independent variable (IV) on change in level-1 dependent variable (DV) by testing the regression of $\mathrm{DV}_{t+1}$ on $\mathrm{IV}_{t}$, controlling for $\mathrm{DV}$ at moment $t\left(\mathrm{DV}_{t}\right)$. Following Delespaul's ${ }^{21}$ guideline as well as previous ESM studies, ${ }^{2,9-11}$ participants who completed less than one-third of total entries (i.e. 20 valid reports) were excluded from the analysis.

To examine whether any of the identified main effects were moderated by patients' diagnostic group or episodic status (i.e. their level2 clinical characteristics), we conducted three sensitivity analyses. For each of the identified main effects, an interaction term between the significant IV and patient's diagnostic group (bipolar type 1 versus type 2 ), an interaction term between the significant IV and patient's episodic status (in an active episode versus not in an active episode) and an interaction term between the significant IV and gender (male versus female) were tested in additional regression models.

To test the role of extreme cognition on the moment-to-moment main effects, we modelled the interaction effects of extreme cognition as level-2 IV and affective states as level-1 $\mathrm{IV}_{t}$ on coping behaviour as $\mathrm{DV}_{t+1}$, controlling for $\mathrm{DV}_{t}$. Data analysis was conducted on Stata version 12 for Windows. Statistical significance was set at $P<0.05$.

\section{Results}

\section{Demographic and clinical data}

Among 125 referrals, 64 fulfilled the inclusion criteria, among whom 46 completed at least 20 ESM entries and were included in data analysis, which is comparable with other recent ESM studies. $^{2,9,11}$ In total, the participants completed 1807 ESM entries, with a mean completion rate of $65.85 \%$ (range $35-100 \%$ ), which was calculated as the number of completed entries divided by the total number of entries expected over the 6-day period.

As shown in Table 1, among the 46 participants, 11 (23.90\%) were males and $35(76.10 \%)$ were females. Average age was 38.93 years (range 18-61). Participants had received psychiatric service for an average of 10.77 years (range $0-40$ ), with a majority of the sample $(n=43,93.48 \%)$ being on psychiatric medication. Thirtythree individuals $(71.73 \%)$ had a diagnosis of bipolar type 1 disorder and $13(28.26 \%)$ had a diagnosis of bipolar type 2 disorder. The numbers of participants who met the DSM-IV criteria for a depressive episode, hypomanic/manic episode or and mixed episode in the past month were five (10.87\%), seven (15.22\%) and seven (15.22\%), respectively.

Baseline mood symptom severity scores were as follows: YMRS = 2.16 (s.d. = 3.90), MADRS $=6.66$ (s.d. = 7.97) and HRSA $=9.32$ (s.d. =8.77). On MADRS, ${ }^{16} 26$ participants $(60.47 \%)$ scored within a normal range (0-6), 13 (30.23\%) scored within a mild range (7-19) and four (9.30\%) sored within a moderate range (20-34). On HRSA, ${ }^{18} 34$ participants $(79.07 \%)$ scored within a mild range (0-17), six (13.95\%) scored within a mild-to-moderate range (18-24) and three (6.98\%) scored within a moderate range (25-30).

Average affect scores across 6 days, as assessed by ESM (score range 1-7), were as follows: negative affect, 2.37 (s.d. = 1.39); positive affect, 4.08 (s.d. = 1.74) and elevated affect, 1.82 (s.d.=1.08). For participants who were not currently in an active episode ( $n=$ 27), average affect scores across 6 days were as follows: negative affect, 2.01 (s.d. = 1.20); positive affect, 4.48 (s.d. = 1.71) and elevated affect, 1.90 (s.d.=1.13). Average coping scores across 6 days, as assessed by ESM (score range 1-7), were as follows: rumination, 2.35 (s.d. =1.62); risk-taking 1.30 (s.d. $=0.78$ ) and adaptive coping 3.16 (s.d. $=1.82$ ).

\section{Between-moment prediction from affective states to coping behaviours}

Moment-to-moment relationships between affective states at time $t$ and coping behaviours at time $t+1$ are shown in Table 2. After controlling for rumination ${ }_{t}$, level of negative affect $_{t}$ predicted an

\begin{tabular}{|c|c|c|c|c|}
\hline Characteristics & $N(\%)$ & Mean (s.d.) & Median & q25 and q75 \\
\hline Age (years) & & $38.93(12.01)$ & 39 & 31,49 \\
\hline \multicolumn{5}{|l|}{ Gender } \\
\hline Male & $11(23.90 \%)$ & & & \\
\hline Female & $35(76.10 \%)$ & & & \\
\hline Education level & & $13.07(3.23)$ & 13 & 11,16 \\
\hline \multicolumn{5}{|l|}{ Bipolar disorder diagnosis } \\
\hline Bipolar type 1 disorder & $33(71.73 \%)$ & & & \\
\hline Bipolar type 2 disorder & $13(28.26 \%)$ & & & \\
\hline Onset of bipolar disorder & & $10.77(10.71)$ & 8.5 & 2,16 \\
\hline$>3$ years & $16(36.36 \%)$ & & & \\
\hline$\leq 3$ years & $28(63.64 \%)$ & & & \\
\hline Number of admissions & & 2.09 (2.71) & 1 & 0,2 \\
\hline \multicolumn{5}{|l|}{ Bipolar episode in the past month } \\
\hline Not in an active episode & $27(58.70 \%)$ & & & \\
\hline Depressive episode & $5(10.87 \%)$ & & & \\
\hline Hypomanic/manic episode & $7(15.22 \%)$ & & & \\
\hline Mixed episode (depressive and hypomanic/manic) & $7(15.22 \%)$ & & & \\
\hline \multicolumn{5}{|l|}{ Baseline mood symptoms } \\
\hline Young Mania Rating Scale & & $2.16(3.90)$ & 0 & 0,2 \\
\hline Montgomery-Asberg Depression Rating Scale & & $6.66(7.97)$ & 2.5 & $0,12.5$ \\
\hline Hamilton Rating Scale for Anxiety & & $9.32(8.77)$ & 6.5 & 2,16 \\
\hline
\end{tabular}


Table 2 Between-moment regression of coping behaviours on affective states (models 1-9) and between-moment regression of affective states on coping behaviours (models 10-18)

\begin{tabular}{|c|c|c|c|c|c|}
\hline Model & Independent variable & Dependent variable & $\beta$ (s.e.) & $95 \% \mathrm{Cl}$ & $P$ value \\
\hline \multirow[t]{2}{*}{1} & Negative affect $t_{t}$ & \multirow[t]{2}{*}{ Risk-taking $_{t+1}$} & $0.07(0.03)$ & $0.01-0.14$ & 0.028 \\
\hline & Risk-taking & & $0.24(0.03)$ & $0.19-0.30$ & $<0.001$ \\
\hline \multirow[t]{2}{*}{2} & Negative affect ${ }_{t}$ & \multirow{2}{*}{ Rumination $_{t+1}$} & $0.21(0.08)$ & $0.05-0.36$ & 0.009 \\
\hline & Rumination $_{t}$ & & $0.22(0.03)$ & $0.16-0.28$ & $<0.001$ \\
\hline \multirow[t]{2}{*}{3} & Negative affect ${ }_{t}$ & \multirow[t]{2}{*}{ Adaptive coping $t_{t+1}$} & $-0.10(0.14)$ & $-0.39-0.18$ & 0.473 \\
\hline & Adaptive coping ${ }_{t}$ & & $0.20(0.03)$ & $0.14-0.26$ & $<0.001$ \\
\hline \multirow[t]{2}{*}{4} & Positive affect $_{t}$ & \multirow[t]{2}{*}{ Risk-taking $t+1$} & $-0.03(0.03)$ & $-0.08-0.02$ & 0.174 \\
\hline & Risk-taking ${ }_{t}$ & & $0.25(0.03)$ & $0.19-0.30$ & $<0.001$ \\
\hline \multirow[t]{2}{*}{5} & Positive affect $_{t}$ & \multirow[t]{2}{*}{ Rumination $_{t+1}$} & $-0.15(0.06)$ & $-0.26--0.03$ & 0.014 \\
\hline & Rumination $_{t}$ & & $0.22(0.03)$ & $0.16-0.28$ & $<0.001$ \\
\hline \multirow[t]{2}{*}{6} & Positive affect ${ }_{t}$ & \multirow[t]{2}{*}{ Adaptive coping $_{t+1}$} & $0.26(0.11)$ & $0.04-0.47$ & 0.018 \\
\hline & Adaptive coping ${ }_{t}$ & & $0.19(0.03)$ & $0.13-0.25$ & $<0.001$ \\
\hline \multirow[t]{2}{*}{7} & Elevated affect $t_{t}$ & \multirow{2}{*}{ Risk-taking ${ }_{t+1}$} & $-0.07(0.04)$ & $-0.15-0.01$ & 0.108 \\
\hline & Risk-taking ${ }_{t}$ & & $0.25(0.03)$ & $0.19-0.30$ & $<0.001$ \\
\hline \multirow[t]{2}{*}{8} & Elevated affect $t_{t}$ & \multirow[t]{2}{*}{ Rumination $_{t+1}$} & $-0.00(0.10)$ & $-0.20-0.19$ & 0.974 \\
\hline & Rumination $_{t}$ & & $0.23(0.03)$ & $0.17-0.29$ & $<0.001$ \\
\hline \multirow[t]{2}{*}{9} & Elevated affect ${ }_{t}$ & \multirow[t]{2}{*}{ Adaptive coping $_{t+1}$} & $0.34(0.18)$ & $-0.01-0.70$ & 0.058 \\
\hline & Adaptive coping & & $0.19(0.03)$ & $0.13-0.25$ & $<0.001$ \\
\hline \multirow[t]{2}{*}{10} & Risk-taking $_{t}$ & \multirow[t]{2}{*}{ Negative affect $_{t+1}$} & $0.02(0.02)$ & $-0.03-0.07$ & 0.414 \\
\hline & Negative affect $t_{t}$ & & $0.21(0.03)$ & $0.16-0.27$ & $<0.001$ \\
\hline \multirow[t]{2}{*}{11} & Risk-taking $_{t}$ & \multirow[t]{2}{*}{ Positive affect $t_{t+1}$} & $0.04(0.03)$ & $-0.02-0.10$ & 0.234 \\
\hline & Positive affect $t_{t}$ & & $0.18(0.03)$ & $0.12-0.23$ & $<0.001$ \\
\hline \multirow[t]{2}{*}{12} & Risk-taking $_{t}$ & \multirow[t]{2}{*}{ Elevated affect $_{t+1}$} & $0.01(0.02)$ & $-0.03-0.04$ & 0.748 \\
\hline & Elevated affect ${ }_{t}$ & & $0.26(0.03)$ & $0.21-0.32$ & $<0.001$ \\
\hline \multirow[t]{2}{*}{13} & Rumination $_{t}$ & \multirow[t]{2}{*}{ Negative affect $t_{t+1}$} & $0.03(0.01)$ & $0.01-0.05$ & 0.009 \\
\hline & Negative affect $t_{t}$ & & $0.20(0.03)$ & $0.15-0.26$ & $<0.001$ \\
\hline \multirow[t]{2}{*}{14} & Rumination $_{t}$ & \multirow[t]{2}{*}{ Positive affect $_{t+1}$} & $-0.03(0.01)$ & $-0.06--0.01$ & 0.016 \\
\hline & Positive affect $t_{t}$ & & $0.17(0.03)$ & $0.12-0.22$ & $<0.001$ \\
\hline 15 & Rumination $_{t}$ & Elevated affect $t_{t+1}$ & $0.00(0.01)$ & $-0.01-0.02$ & 0.843 \\
\hline & Elevated affect $t_{t}$ & & $0.26(0.03)$ & $0.21-0.32$ & $<0.001$ \\
\hline 16 & Adaptive coping $t$ & Negative affect $t_{t+1}$ & $-0.01(0.01)$ & $-0.02-0.01$ & 0.319 \\
\hline & Negative affect $t_{t}$ & & $0.21(0.03)$ & $0.16-0.27$ & $<0.001$ \\
\hline 17 & Adaptive coping ${ }_{t}$ & Positive affect $t_{t+1}$ & $0.02(0.01)$ & $0.00-0.03$ & 0.019 \\
\hline & Positive affect ${ }_{t}$ & & $0.17(0.03)$ & $0.12-0.23$ & $<0.001$ \\
\hline 18 & Adaptive coping ${ }_{t}$ & Elevated affect $t_{t+1}$ & $0.02(0.00)$ & $0.01-0.02$ & $<0.001$ \\
\hline & Elevated affect $t_{t}$ & & $0.26(0.03)$ & $0.20-0.31$ & $<0.001$ \\
\hline
\end{tabular}

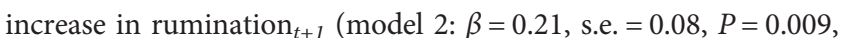
95\% CI 0.05-0.36) and level of positive affect $_{t}$ predicted a decrease

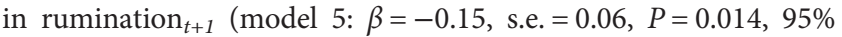
$\mathrm{CI}-0.26$ to -0.03$)$. After controlling for risk-taking, level of negative affect $_{t}$ predicted an increase in risk taking $_{t+1}$ (model 1: $\beta=0.07$, s.e. $=0.03, P=0.028,95 \%$ CI 0.01-0.14). After controlling for adaptive coping ${ }_{t}$, level of positive affect $_{t}$ predicted an increase in adaptive coping $_{t+1}$ (model 6: $\beta=0.26$, s.e. $=0.11, P=0.018,95 \%$ CI 0.04-0.47).

Sensitivity analysis of diagnostic group revealed that the effect of positive affect on decrease in rumination $_{t+1}$ was smaller in the bipolar type 2 group than the bipolar type 1 group $(\beta=-0.30$, s.e. $=0.14, P=$ $0.034,95 \% \mathrm{CI}-0.58$ to -0.02 ). The other main effects from affective states to coping behaviours did not significantly differ across other diagnostic groups, episodic status or gender (all $P>0.05$ ).

\section{Between-moment prediction from coping behaviours to affective states}

Moment-to-moment relationships between coping behaviours at time $t$ and affective states at time $t+1$ are shown in Table 2. After con-

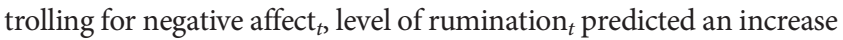
in negative affect $_{t+1}$ (model 13: $\beta=0.03$, s.e. $=0.01, P=0.009,95 \% \mathrm{CI}$ 0.01-0.05). After controlling for positive affect $_{t}$, level of rumination r $_{t}$ predicted a decrease in positive affect $_{t+1}$ (model $14: \beta=-0.03$, s.e. $=$ $0.01, P=0.016,95 \%$ CI -0.06 to -0.01 ), whereas level of adaptive

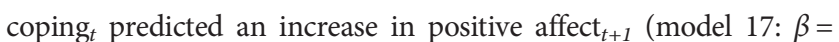
0.02 , s.e. $=0.01, P=0.019,95 \%$ CI $0.00-0.03$ ). After controlling for elevated affect ${ }_{t}$, level of adaptive coping ${ }_{t}$ predicted an increase in elevated affect $_{t+1}$ (model 18: $\beta=0.02$, s.e. $=0.00, P<0.001,95 \%$ CI $0.01-$ $0.02)$.

Sensitivity analysis of episodic status revealed that the effect of rumination $_{t}$ on decrease in positive affect $_{t+1}(\beta=-0.07$, s.e. $=0.02$, $P<0.001,95 \%$ CI -0.11 to -0.03$)$ and the effect of adaptive coping $_{t}$ on increase in elevated affect ${ }_{t+1}(\beta=-0.03$, s.e. $=0.01, P<$ $0.001,95 \%$ CI -0.04 to -0.02 ) were weaker in individuals who were in an active episode than individuals who were not in active episode. The other main effects from coping behaviours to affective states did not significantly differ across diagnostic groups, other episodic status or gender (all $P>0.05$ ).

Figure 1 summarises the main effects of cross-moment relationships between affective states and coping behaviours in a schematic manner.

Role of baseline level of extreme cognitive appraisals on the moment-to-moment relationship between affective states and coping behaviours

As shown in Table 3, time-lagged analysis demonstrated that all five factor scores of HAPPI predicted a stronger increase in rumination ${ }_{t+1}$ following negative affect $_{t}$ (models $2 \mathrm{a}-2 \mathrm{e}$ ), whereas all factors except factor IV of HAPPI predicted a more marked decrease in 


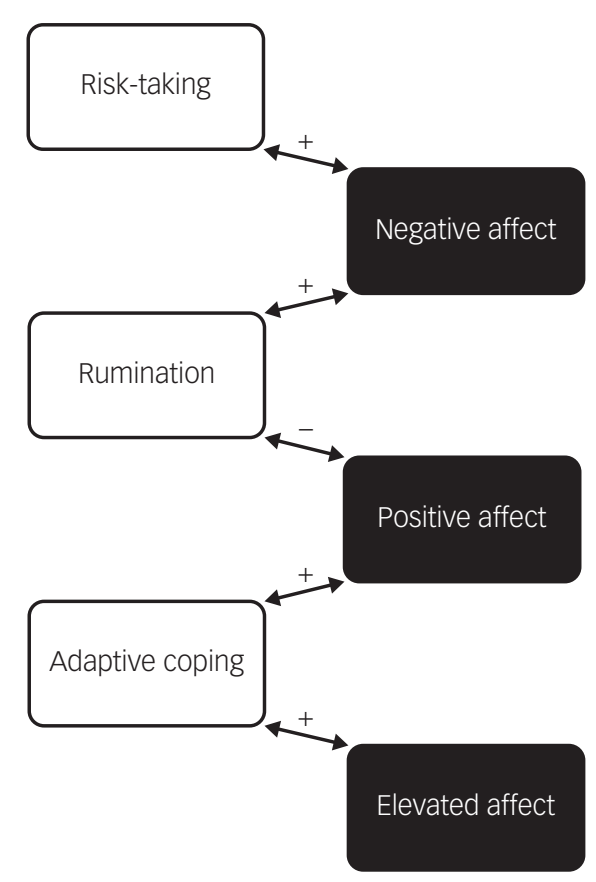

Fig. 1 Schematic diagram illustrating time-lagged relationships between affective states and coping behaviours.

rumination $_{t+1}$ following positive affect ${ }_{t}$ (models $5 \mathrm{a}-5 \mathrm{~d}$ ). All five factors of HAPPI predicted a stronger increase in adaptive coping ${ }_{t}$ ${ }_{+1}$ following positive affect $t_{t}$ (models $6 \mathrm{a}-6 \mathrm{e}$ ). Three factors predicted a stronger increase in risk taking ${ }_{t+1}$ following negative affect $_{t}$ (models $1 \mathrm{a}-1 \mathrm{c})$.

\section{Discussion}

In this study, we modelled moment-to-moment interaction between various affective states and coping behaviours and tested the role of cognitive appraisals on these moment-to-moment dynamics among 46 individuals with bipolar disorder. By using a fine-grained experience sampling assessment, we delineated how one variable drives subsequent changes in another variable and captured the intricate relationships between affect and behaviour in a naturalistic manner. Major findings are as follows: (a) negative affect predicted an increase in rumination in the next assessment time point, and vice versa; (b) positive affect predicted a decrease in rumination and an increase in adaptive coping in the next assessment time point, and vice versa and (c) extreme cognitive appraisals predicted stronger associations between positive and negative affective states and coping behaviours.

Coping behaviours are carried out in an attempt to control or regulate affective symptoms. ${ }^{1}$ Although ascent behaviours (such as risk-taking and alcohol use) contribute to increases in activation levels, descent behaviours (such as rumination and social withdrawal) contribute to decreases in activation levels. In the current study, we found a feedback loop between rumination and affective states, whereby individuals ruminated more after experiencing negative affect and less after experiencing positive affect. The vicious cycle became self-perpetuating as rumination in turn led to more negative affect and less positive affect. Our results, together with Pavlickova et $a l^{2}$ and the depression literature, suggest that rumination maintains negative emotions in bipolar disorder in a similar way as in unipolar depression. As rumination contributes to maintenance of disruptive affective disturbance in individuals with bipolar disorder, our result raises the possibility of using rumination-based interventions, which have proven efficacy in major depressive disorder, ${ }^{22}$ for treating emotion disturbances in bipolar disorder.

We included separate ESM items for assessing elevated affect and positive affect. Rather than combining these items into one factor as in Pavlickova et al, ${ }^{2}$ our measures allowed us to differentiate the specific relationship between coping behaviours with an elevated (hypo)manic state as opposed to a relaxed/content state. When assessing elevated affect and positive affect separately, the association between risk-taking and positive affect reported by Pavlickova et $a l^{2}$ was no longer present. Instead, our results revealed that positive affect (i.e. a relaxed/content state) predicted subsequent engagement in adaptive coping. The positive feedback loop between positive affect and adaptive coping suggests that engaging in adaptive coping rather than rumination is helpful for eliciting positive affective experiences in individuals with bipolar disorder.

Contrary to our hypothesis, risk-taking did not predict any affective states. The lack of association may be partly explained by a generally low level of risk-taking in our sample (mean ESM score, 1.30). Although negative affect predicted risk-taking, we take caution in interpreting this finding as the effect was small and preliminary.

Our sensitivity analyses revealed that none of the eight crossmoment associations between affective states and coping behaviours differed across genders. Only one cross-moment association significantly differed between diagnostic group (i.e. the effect of positive affect ${ }_{t}$ on decrease in rumination trl $_{1}$ was smaller in the bipolar type 2 group than the bipolar type 1 group), and two differed between patients who were versus were not in an active episode (i.e. the effect of rumination on decrease in positive affect $_{t+1}$ and the effect of adaptive coping $t$ on increase in elevated affect ${ }_{t+1}$ were weaker among individuals who were in an active episode than individuals who were not in active episode). Therefore, the moment-to-moment relationships between affective states and coping behaviours were relatively robust and were not totally explained by diagnosis or clinical status. On speculation of the regression coefficients, it is tempting to argue that effects from affective states to coping behaviours are stronger than the effects from coping behaviours to affective states. However, as the overall effect sizes are small, this claim cannot be conclusive until our findings are replicated.

Extreme appraisals of internal states catastrophise the meaning of internal states, leading to engagement in maladaptive coping behaviours among individuals with bipolar disorder. ${ }^{1}$ This theoretical claim was partially supported by our data. For example, individuals who scored high on 'success activation and triumph over fear' (factor IV) tended to cope with negative affect by seeking sensation (e.g. alcohol use and reckless behaviours). This might lend support to the argument that risk-taking behaviour is adopted to restore individuals' sense of self and to lower their level of anxiety and depression. ${ }^{23}$ However, since cognitive appraisals exacerbated the positive cycle between positive affect and adaptive coping as well, it is also possible that individuals with more extreme cognitive appraisals are more likely to cope with their affective states in general, regardless of emotional valence.

\section{Limitations}

There were several limitations to our study. First, although our sample was representative of individuals with bipolar disorder in an out-patient setting, it is hard to evaluate to what extent heterogeneity in clinical characteristics (including duration of illness, medication regime and comorbidity etc.) would have affected the 
Table 3 Moderation effects of cognitive appraisal styles on the moment-to-moment relationships from affective states to coping behaviours

\begin{tabular}{|c|c|c|c|c|c|}
\hline Model & Independent variable & Dependent variable & $\beta$ (s.e.) & $95 \% \mathrm{Cl}$ & $P$ value \\
\hline \multirow[t]{4}{*}{$1 \mathrm{a}$} & Negative affect $t_{t}$ & Risk-taking $g_{t+1}$ & $0.07(0.03)$ & $0.01-0.14$ & 0.028 \\
\hline & Risk-taking & & $0.24(0.03)$ & $0.19-0.30$ & $<0.001$ \\
\hline & HAPPI factor $1 \times$ negative affect $_{t}$ & & $0.00(0.00)$ & $-0.00-0.00$ & 0.049 \\
\hline & HAPPI factor $1 \times$ risk-taking & & $0.24(0.03)$ & $0.18-0.30$ & $<0.001$ \\
\hline \multirow[t]{4}{*}{$1 b$} & Negative affect ${ }_{t}$ & Risk-taking $g_{t+1}$ & $0.07(0.03)$ & $0.01-0.14$ & 0.028 \\
\hline & Risk-taking & & $0.24(0.03)$ & $0.19-0.30$ & $<0.001$ \\
\hline & HAPPI factor $3 \times$ negative affect $_{t}$ & & $0.00(0.00)$ & $0.00-0.00$ & 0.027 \\
\hline & HAPPI factor $3 \times$ risk-taking ${ }_{t}$ & & $0.24(0.03)$ & $0.19-0.30$ & $<0.001$ \\
\hline \multirow[t]{4}{*}{$1 \mathrm{C}$} & Negative affect $t$ & Risk-taking $g_{t+1}$ & $0.07(0.03)$ & $0.01-0.14$ & 0.028 \\
\hline & Risk-taking $t$ & & $0.24(0.03)$ & $0.19-0.30$ & $<0.001$ \\
\hline & HAPPI factor $4 \times$ negative affect $t$ & & $0.00(0.00)$ & $0.00-0.00$ & 0.038 \\
\hline & HAPPI factor $4 \times$ risk-taking & & $0.24(0.03)$ & $0.20-0.30$ & $<0.001$ \\
\hline \multirow[t]{4}{*}{$2 a$} & Negative affect ${ }_{t}$ & Rumination $_{t+1}$ & $0.21(0.08)$ & $0.05-0.36$ & 0.009 \\
\hline & Rumination $_{t}$ & & $0.22(0.03)$ & $0.16-0.28$ & $<0.001$ \\
\hline & HAPPI factor $1 \times$ negative affect $_{t}$ & & $0.00(0.00)$ & $0.00-0.01$ & 0.003 \\
\hline & HAPPI factor $1 \times$ rumination $_{t}$ & & $0.22(0.03)$ & $0.16-0.28$ & $<0.001$ \\
\hline \multirow[t]{4}{*}{$2 b$} & Negative affect $t_{t}$ & Rumination $_{t+1}$ & $0.21(0.08)$ & $0.05-0.36$ & 0.009 \\
\hline & Rumination $_{t}$ & & $0.22(0.03)$ & $0.16-0.28$ & $<0.001$ \\
\hline & HAPPI factor $2 \times$ negative affect $_{t}$ & & $0.01(0.00)$ & $0.00-0.01$ & 0.002 \\
\hline & HAPPI factor $2 \times$ rumination $_{t}$ & & $0.22(0.03)$ & $0.16-0.28$ & $<0.001$ \\
\hline \multirow[t]{4}{*}{$2 \mathrm{C}$} & Negative affect $t_{t}$ & Rumination $_{t+1}$ & $0.21(0.08)$ & $0.05-0.36$ & 0.009 \\
\hline & Rumination $_{t}$ & & $0.22(0.03)$ & $0.16-0.28$ & $<0.001$ \\
\hline & HAPPI factor $3 \times$ negative affect $_{t}$ & & $0.00(0.00)$ & $0.00-0.01$ & 0.014 \\
\hline & HAPPI factor $3 \times$ rumination $_{t}$ & & $0.22(0.03)$ & $0.16-0.28$ & $<0.001$ \\
\hline \multirow[t]{4}{*}{$2 d$} & Negative affect $_{t}$ & Rumination $_{t+1}$ & $0.21(0.08)$ & $0.05-0.36$ & 0.009 \\
\hline & Rumination $_{t}$ & & $0.22(0.03)$ & $0.16-0.28$ & $<0.001$ \\
\hline & HAPPI factor $4 \times$ negative affect $_{t}$ & & $0.00(0.00)$ & $0.00-0.01$ & 0.019 \\
\hline & HAPPI factor $4 \times$ rumination $_{t}$ & & $0.22(0.03)$ & $0.16-0.28$ & $<0.001$ \\
\hline \multirow[t]{4}{*}{$2 e$} & Negative affect ${ }_{t}$ & Rumination $_{t+1}$ & $0.21(0.08)$ & $0.05-0.36$ & 0.009 \\
\hline & Rumination $_{t}$ & & $0.22(0.03)$ & $0.16-0.28$ & $<0.001$ \\
\hline & HAPPI factor $5 \times$ negative affect $t$ & & $0.00(0.00)$ & $0.00-0.01$ & 0.030 \\
\hline & HAPPI factor $5 \times$ rumination $_{t}$ & & $0.22(0.03)$ & $0.16-0.28$ & $<0.001$ \\
\hline \multirow[t]{4}{*}{$5 a$} & Positive affect $t_{t}$ & Rumination $_{t+1}$ & $-0.15(0.06)$ & $-0.26--0.03$ & 0.014 \\
\hline & Rumination $_{t}$ & & $0.22(0.03)$ & $0.16-0.28$ & $<0.001$ \\
\hline & HAPPI factor $1 \times$ positive affect $_{t}$ & & $-0.00(0.00)$ & $-0.01-0.00$ & 0.001 \\
\hline & HAPPI factor $1 \times$ rumination $_{t}$ & & $0.23(0.03)$ & $0.17-0.29$ & $<0.001$ \\
\hline \multirow[t]{4}{*}{$5 b$} & Positive affect $t_{t}$ & Rumination $_{t+1}$ & $-0.15(0.06)$ & $-0.26--0.03$ & 0.014 \\
\hline & Rumination $_{t}$ & & $0.22(0.03)$ & $0.16-0.28$ & $<0.001$ \\
\hline & HAPPI factor $2 \times$ positive affect $_{t}$ & & $-0.01(0.00)$ & $-0.01-0.00$ & $<0.001$ \\
\hline & HAPPI factor $2 \times$ rumination $_{t}$ & & $0.22(0.03)$ & $0.16-0.28$ & $<0.001$ \\
\hline \multirow[t]{4}{*}{$5 c$} & Positive affect $t_{t}$ & Rumination $_{t+1}$ & $-0.15(0.06)$ & $-0.26--0.03$ & 0.014 \\
\hline & Rumination $_{t}$ & & $0.22(0.03)$ & $0.16-0.28$ & $<0.001$ \\
\hline & HAPPI factor $3 \times$ positive affect $_{t}$ & & $-0.00(0.00)$ & $-0.01-0.00$ & 0.029 \\
\hline & HAPPI factor $3 \times$ rumination $_{t}$ & & $0.22(0.03)$ & $0.16-0.28$ & $<0.001$ \\
\hline \multirow[t]{4}{*}{$5 d$} & Positive affect $t_{t}$ & Rumination $_{t+1}$ & $-0.15(0.06)$ & $-0.26--0.03$ & 0.014 \\
\hline & Rumination $_{t}$ & & $0.22(0.03)$ & $0.16-0.28$ & $<0.001$ \\
\hline & HAPPI factor $5 \times$ positive affect $_{t}$ & & $-0.00(0.00)$ & $-0.01-0.00$ & 0.009 \\
\hline & HAPPI factor $5 \times$ rumination $_{t}$ & & $0.22(0.03)$ & $0.16-0.28$ & $<0.001$ \\
\hline $6 a$ & Positive affect $t_{t}$ & Adaptive coping $t_{t+1}$ & $0.26(0.11)$ & $0.04-0.47$ & 0.018 \\
\hline & Adaptive copingt & & $0.19(0.03)$ & $0.13-0.25$ & $<0.001$ \\
\hline & HAPPI factor $1 \times$ positive affect $_{t}$ & & $0.01(0.00)$ & $0.01-0.01$ & 0.002 \\
\hline & HAPPI factor $1 \times$ adaptive coping $t$ & & $0.20(0.03)$ & $0.14-0.26$ & $<0.001$ \\
\hline $6 b$ & Positive affect $t_{t}$ & Adaptive coping $g_{t+1}$ & $0.26(0.11)$ & $0.04-0.47$ & 0.018 \\
\hline & Adaptive coping & & $0.19(0.03)$ & $0.13-0.25$ & $<0.001$ \\
\hline & HAPPI factor $2 \times$ positive affect $_{t}$ & & $0.01(0.00)$ & $0.01-0.01$ & 0.009 \\
\hline & HAPPI factor $2 \times$ adaptive coping $t$ & & $0.20(0.03)$ & $0.14-0.26$ & $<0.001$ \\
\hline $6 c$ & Positive affect $t_{t}$ & Adaptive coping $t_{t+1}$ & $0.26(0.11)$ & $0.04-0.47$ & 0.018 \\
\hline & Adaptive copingt & & $0.19(0.03)$ & $0.13-0.25$ & $<0.001$ \\
\hline & HAPPI factor $3 \times$ positive affect $_{t}$ & & $0.01(0.00)$ & $0.00-0.01$ & 0.008 \\
\hline & HAPPI factor $3 \times$ adaptive coping $t$ & & $0.20(0.03)$ & $0.14-0.26$ & $<0.001$ \\
\hline $6 d$ & Positive affect $t_{t}$ & Adaptive coping $g_{t+1}$ & $0.26(0.11)$ & $0.04-0.47$ & 0.018 \\
\hline & Adaptive copingt & & $0.19(0.03)$ & $0.13-0.25$ & $<0.001$ \\
\hline & HAPPI factor $4 \times$ positive affect $_{t}$ & & $0.01(0.00)$ & $0.00-0.01$ & 0.025 \\
\hline & HAPPI factor $4 \times$ adaptive coping $t$ & & $0.20(0.03)$ & $0.14-0.26$ & $<0.001$ \\
\hline $6 e$ & Positive affect $t_{t}$ & Adaptive coping $_{t+1}$ & $0.26(0.11)$ & $0.04-0.47$ & 0.018 \\
\hline & Adaptive coping & & $0.19(0.03)$ & $0.13-0.25$ & $<0.001$ \\
\hline & 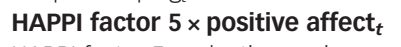 & & $0.01(0.00)$ & $0.00-0.01$ & 0.042 \\
\hline & HAPPI factor $5 \times$ adaptive coping & & $0.19(0.03)$ & $0.13-0.25$ & $<0.001$ \\
\hline
\end{tabular}


results. Second, the current study was also limited by a lack of a control group. A healthy control group could help us discern the extent to which the association between affective states and coping behaviours is specific to the bipolar disorder group. A psychiatric control group that had heightened emotionality (e.g. pathological gamblers, or individuals with major depressive disorder or borderline personality disorder) would also be a welcomed addition to this area of research. Third, as the ESM signal scheduling was generated by a computer program in a pseudo-random manner, the duration of time-lag varied between days and persons. Therefore, we could not conclude how long it took for the moment-to-moment associations to occur. Fourth, our ESM completion rate was $65 \%$, which was relatively low when compared with previous studies. Finally, we considered endorsement of the Response Style Questionnaire ${ }^{14}$ items as representing behavioural coping. It is possible that some of the items might happen infrequently or are formulated in a rather extreme way, hence explaining the relatively low endorsement rate. More importantly, unless we explicitly ask the individuals for the reason of their behaviours, it remained an assumption that individuals engage in behaviours such as risk-taking or rumination so as to cope with their affective states. As newer coping strategies such as acceptance and tolerance under the mindfulness conceptualisation have gained increased attention in the field and have been found to be effective in reducing symptom severity, ${ }^{24}$ additional coping strategies could be included in future research.

\section{Clinical implications}

ESM supplements traditional assessment methods, and is particularly well suited for assessing subjective experiences of a fluctuating nature. These individually generated ESM data may offer potentially useful information for psychoeducation and intervention. As has been shown in treatment for depression, ${ }^{25}$ associations between emotional states and behaviour as captured by ESM can be discussed between the patient and therapist in the context of functional analysis and coping enhancement within the cognitive behavioural therapy framework. Moreover, if the effect of extreme cognitive appraisals on affective symptoms and coping behaviours is replicated, this could shed light on targeting cognitive appraisals as a potential therapeutic mechanism for psychological treatment for bipolar disorder.

Michelle Hoi-ting Leung, MSSC, MPsyMed, PgDip, Clinical Psychology trainee, Department of Psychology, The Chinese University of Hong Kong, Hong Kong SAR, China: Suzanne Ho-wai So, PhD, MSSc, Associate Professor, Department of Psychology, The Chinese University of Hong Kong, Hong Kong SAR, China; Nate Tsz-Kit Kwok, MSSC, Clinical Psychologist, Department of Psychology, The Chinese University of Hong Kong, Hong Kong SAR, China; Iris Hoi-ching Ng, MSSC, Clinical Psychology trainee, Department of Psychology, The Chinese University of Hong Kong, Hong Kong SAR, China; Pui-shuen Chan, MSSc, Clinical Psychology trainee, Department of Psychology, The Chinese University of Hong Kong, Hong Kong SAR, China; Chloe Chor-wing Lo, MSSC, Clinical Psychology trainee, Department of Psychology, The Chinese University of Hon Kong Hong Kong SAR, China; Shirley Na MSSC, Clinical Psychology trainee, Department of Psychology, The Chinese University of Hong Kong, Hong Kong SAR, China; Arthur Dun-ping Mak, MBChB, FRCPsych, Associate Professor, Department of Psychiatry, The Chinese University of Hong Kong, Hong Kong SAR, China; Sing Lee, FHKAM, FRCPsych(UK), MBBS, Professor, Department of Psychiatry, The Chinese University of Hong Kong, Hong Kong SAR, China

Correspondence: Dr Suzanne So, Room 321, Wong Foo Yuan Building, Department of Psychology, The Chinese University of Hong Kong, New Territories, Hong Kong SAR, China.Email: shwso@psy.cuhk.edu.hk

First received 19 Oct 2018, final revision 10 Apr 2019, accepted 26 Apr 2019

\section{Funding}

S.H.S. is supported by the Croucher Foundation Startup Fund.
Appendix Experience sampling methodology items on engagement in coping behaviour (based on Nolen-Hoeksema's Response Style

Questionnaire

'Between this beep and the previous beep, how much have you engaged in the following behaviour?'

1. Make a plan to overcome a problem

2. Talk it out with someone whose opinions you respect (friend, clergy, family, etc.)

3. Seek out and engage in casual sexual relations

4. Think about how sad you feel

5. Do something enjoyable

6. Drink alcohol excessively

7. Think about how alone you feel

8. Think 'I'm going to do something to make myself feel better'

9. Isolate yourself and think about the reasons why you feel sad

10. Do something reckless or dangerous

1 (not at all) - 7 (very much)

1 (not at all) -7 (very much)

1 (not at all) - 7 (very much)

1 (not at all) -7 (very much)

1 (not at all) -7 (very much)

1 (not at all) -7 (very much)

1 (not at all) -7 (very much)

1 (not at all) -7 (very much)

1 (not at all) -7 (very much)

1 (not at all) - 7 (very much)

Items 1, 2, 5 and 8 represent adaptive coping; items 3,6 and 10 represent risk-taking; and items 4,7 and 9 represent rumination.

\section{References}

1 Mansell W, Morrison A, Reid G, Lowens I, Tai S. The interpretation of, and responses to, changes in internal states: an integrative cognitive model of mood swings and bipolar disorders. Behav Cogn Psychother 2007; 35: 515-39.

2 Pavlickova H, Varese F, Smith A, Myin-Germeys I, Turnbull OH, Emsley R, et al. The dynamics of mood and coping in bipolar disorder: Iongitudinal investigations of the inter-relationship between affect, self-esteem and response styles. PLOS One 2013; 8: e62514.

3 Brans K, Koval P, Verduyn P, Lim YL, Kuppens P. The regulation of negative and positive affect in daily life. Emotion 2013; 13: 926-39.

4 Depue RA, Morrone-Strupinsky JV. Affiliative bonding as a dynamical process: a view from ethology. Behav Brain Sci 2005; 28: 355-6.

5 Johnson SL. Mania and dysregulation in goal pursuit: a review. Clin Psychol Rev 2005; 25: 241-62.

6 GilbertP, McKwan K, Mitra R, Richter A, Franks L, Mills A, et al. An exploration of different types of positive affect in students and patients with a bipolar disorder. Clin Neuropsychiatry 2009; 6: 135-43.

7 Palmier-Claus JE, Dodd A, Tai S, Emsley R, Mansell W. Appraisals to affect: testing the integrative cognitive model of bipolar disorder. Br J Clin Psychol 2015; 55: 225-35.

8 Havermans R, Nicolson NA, Devries MW. Daily hassles, uplifts, and time use in individuals with bipolar disorder in remission. J Nerv Ment Dis 2007; 195: 745-51.

9 Chan Y, So SH, MakADP, Siah KTH, Chan W, WuJ. The temporal relationship of daily life stress, emotions and bowel symptoms in irritable bowel syndrome - diarrhea subtype: a smartphone-based experience sampling study. Neurogastroenterol Motil 2019; 31: e13514.

10 So SH, Chau AKC, Peters E, Swendsen J, Garety P, Kapur S. Moment-to-moment associations between emotional disturbances, aberrant salience, and persecutory delusions. Cogn Neuropsychiatry 2018; 23: 299-306.

11 Tyler E, Jones S, Black N, Carter LA, Barrowclough C. The relationship between bipolar disorder and cannabis use in daily life: an experience sampling study. PLOS One 2015; 10: e0118916.

12 Myin-Germeys I, Birchwood M, Kwapil T. From environment to therapy in psychosis: a real-world momentary assessment approach. Schizophr Bull 2011; 37: 244-7.

13 So SH, Peters ER, Swendsen J, Garety PA, Kapur S. Detecting improvements in acute psychotic symptoms using experience sampling methodology. Psychiatry Res 2013; 210: 82-8.

14 Nolen-Hoeksema S. Responses to depression and their effects on the duration of depressed mood. J Abnorm Psychol 1991; 100: 569-82.

15 So E, Kam I, Leung CM, Chung D, Liu Z, Fong S. The Chinese-bilingual SCID-I/P project: stage 1-reliability for mood disorders and schizophrenia. Hong Kong J Psychiatry 2003; 13: 7-18.

16 Montgomery SA, Asberg M. A new depression scale designed to be sensitive to change. Br J Psychiatry 1979; 134: 382-9.

17 Young RC, Biggs JT, Ziegler VE, Meyer DA. A rating scale for mania: reliability, validity and sensitivity. Br J Psychiatry 1978; 133: 429-35. 
18 Hamilton M. The assessment of anxiety states by rating. Br J Med Psychol 1959; 32: $50-5$.

19 Mansell W, Rigby Z, Tai S, Lowe C. Do current beliefs predict hypomanic symptoms beyond personality style? Factor analysis of the hypomanic attitudes and positive predictions inventory (HAPPI) and its association with hypomanic symptoms in a student population. J Clin Psychol 2008; 64: 1-16.

20 Goldstein H. Multilevel Models in Educational and Social Research. Oxford University Press, 1987

21 Delespaul P. Assessing Schizophrenia in Daily Life: The Experience Sampling Method. Universitaire Pers Maastricht, 1995.

22 Teismann T, Brachel RV, Hanning S, Grillenberger M, Hebermehl L, Hornstein I, et al. A randomized controlled trial on the effectiveness of a ruminationfocused group treatment for residual depression. Psychother Res 2014; 24 : 80-90.

23 Mansell W. The hypomanic attitudes and positive predictions inventory (HAPPI): a pilot study to select cognitions that are elevated in individuals with bipolar disorder compared to non-clinical controls. Behav Cogn Psychother 2006; 34: $467-76$.

24 Chambers R, Gullone E, Allen NB. Mindful emotion regulation: an integrative review. Clin Psychol Rev 2009; 29: 560-72.

25 van Os J, Verhagen S, Marsman A, Peeters F, Bak M, Marcelis M, et al. The experience sampling method as an mHealth tool to support self-monitoring, self-insight, and personalized health care in clinical practice. Depress Anxiety 2017; 34: 481-93.

26 Knowles R, Tai S, Christensen I, Bentall R. Coping with depression and vulnerability to mania: A factor analytic study of the Nolen-Hoeksema (1991) Response Styles Questionnaire. Br J Clin Psychol 2005; 44: 99-112. 